\title{
Maternal D intakes during pregnancy are associated with childhood asthma at 10 years of age
}

\author{
K. Allan ${ }^{1}$, L. C. A. Craig ${ }^{1}$, G. McNeill ${ }^{1}$, S. W. Turner ${ }^{2}$, N. Prabhu ${ }^{2}$, R. Cutts ${ }^{2}$, P. J. Helms ${ }^{2}$, A. Seaton ${ }^{1}$ \\ and G. Devereux ${ }^{2}$ \\ ${ }^{1}$ Public Health Nutrition Research Group, Section of Population Health and ${ }^{2}$ Department of Child Health, Royal Aberdeen \\ Children's Hospital, University of Aberdeen, Foresterhill, Aberdeen AB25 2ZD, UK
}

It has been hypothesised that changing diet has contributed to the recent increase in asthma in westernised countries. The prospective SEATON cohort comprising 2000 pregnant women was established between October 1997 and April 1999 to test the hypothesis that maternal nutrition during pregnancy affects the likelihood of children developing asthma ${ }^{(1)}$.

At 32 weeks gestation mothers' habitual dietary intakes were assessed by FFQ (Version 5.4 Scottish Collaborative Group). One thousand, nine hundred and twenty-four live singletons comprised the birth cohort that has been followed up at 2, 5 and 10 years. Children's asthmatic status was assessed by International Study of Asthma and Allergies in Childhood (ISAAC) questionnaire and their dietary intakes by FFQ (Version C2 Scottish Collaborative Group) ${ }^{(1)}$.

Logistic regression models examined the effects of maternal energy adjusted nutrient intakes while adjusting for potential confounders at 10 years. Analyses focused on vitamins E and D in order to test the hypothesis that previous associations reported at 5 years ${ }^{(1,2)}$ persist into later childhood. OR presented compare highest to lowest quintiles of maternal intake.

The response rate at 10 years was $49 \%$. Contrary to the 5 -year findings ${ }^{(1)}$, at 10 years maternal vitamin E intake during pregnancy was not associated with asthma outcomes cross-sectionally, but maternal vitamin D intake was associated with decreased odds of 'doctor diagnosed asthma', 'wheeze ever' and 'wheeze in the last year' in the children (Table 1).

\begin{tabular}{|c|c|c|c|}
\hline Outcome & $N$ & OR $(95 \% \mathrm{CI})$ & $P$ for Trend \\
\hline Wheeze ever & 733 & $0.46(0.25-0.83)$ & 0.005 \\
\hline Wheeze in last year & 733 & $0.60(0.26-1.38)$ & 0.022 \\
\hline Dr diagnosed asthma & 727 & $0.46(0.34-0.94)$ & 0.023 \\
\hline
\end{tabular}

In conclusion, reduced maternal vitamin D intake during pregnancy is associated with an increased likelihood of childhood asthma at the age of 10 years which is consistent with associations at 5 years, whereas vitamin $\mathrm{E}$ was associated with asthma outcomes at earlier time points but not at 10 years. Intervention trials are required to ascertain if intervention during pregnancy actually reduces the likelihood of childhood asthma.

1. Devereux G, Turner SW, Craig LCA et al. (2006) Low maternal vitamin E intake during pregnancy is associated with asthma in 5-year-old children. Am J Respir Crit Care Med 174, 499-507.

2. Devereux G, Litonjua AA, Turner SW et al. (2007) Maternal vitamin D intake during pregnancy and early childhood wheezing. Am J Clin Nutr 85, 853-9. 